Tecno Lógicas

ISSN 0123-7799

Vol. 19, No. 36, pp. 63-76

Enero-junio de 2016

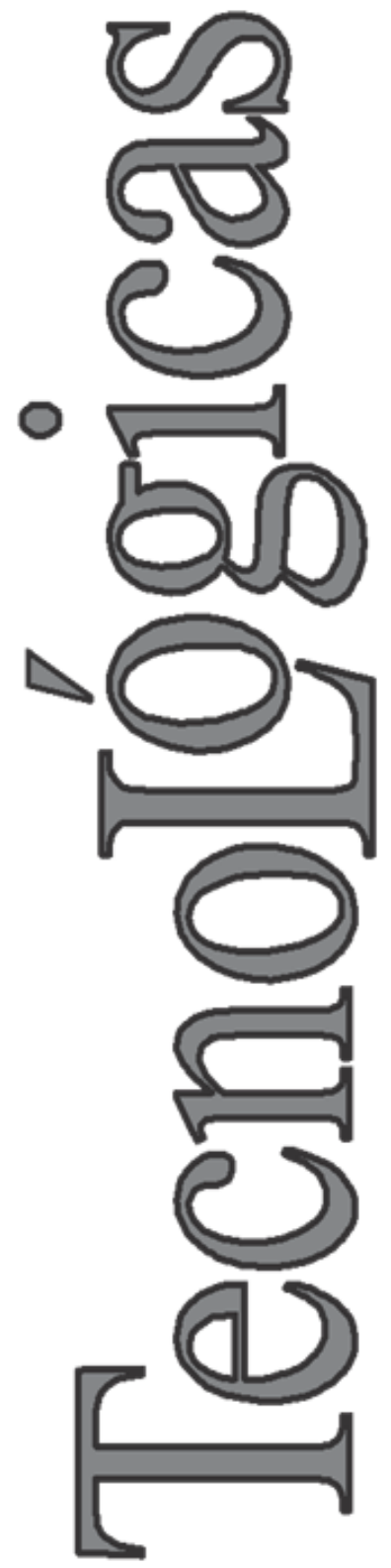

(C) Copyright 2015 por autores y Tecno Lógicas Este trabajo está licenciado bajo una Licencia Internacional Creative Commons Atribución (CC BY)

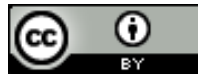

\section{Control repetitivo impar de alto orden de un rectificador monofásico: operación a frecuencia variable}

\section{Odd harmonic high order repetitive control of single-phase pmw rectifiers: varying frequency operation}

\author{
Germán A. Ramos-Fuentes ${ }^{1}$, Iván D. Melo-Lagos² y \\ Fernando J. Regino-Ubarnes ${ }^{3}$
}

Recibido: 18 de junio de 2015, Aceptado: 20 de diciembre de 2015

Cómo citar / How to cite

G.A. Ramos-Fuentes, I.D. Melo-Lagos y F.J. Regino-Ubarnes, "Control repetitivo impar de alto orden de un rectificador monofásico: operación a frecuencia variable”, Tecno Lógicas, vol. 19, no. 36, pp. 63-76, 2015.

1 PhD. en Automática, Departamento de Ingeniería Eléctrica y Electrónica, Facultad de Ingeniería, Universidad Nacional de Colombia, Bogotá-Colombia, garamosf@unal.edu.co

2 Ingeniero Eléctrico, Departamento de Ingeniería Eléctrica y Electrónica, Facultad de Ingeniería, Universidad Nacional de Colombia, Bogotá-Colombia, idmelol@unal.edu.co

3 Ingeniero Eléctrico, Departamento de Ingeniería Eléctrica y Electrónica, Facultad de Ingeniería, Universidad Nacional de Colombia, Bogotá-Colombia, fjreginou@unal.edu.co 


\title{
Resumen
}

Los rectificadores de potencia controlados son dispositivos utilizados con el fin de minimizar la contaminación armónica producida en las redes eléctricas durante el proceso de conversión de la potencia. El objetivo es obtener una corriente de entrada sinusoidal en fase con el voltaje de la red. Aunque es extenso el uso del control Proporcional Integral en el lazo de corriente, no es posible lograr altos desempeños con estos compensadores. Por otro lado, el Control Repetitivo y el Control Resonante son técnicas mediante las cuales se logran resultados excepcionales. La desventaja de estas estrategias de control es la gran pérdida de desempeño en el sistema cuando la frecuencia de la red se desvía de su valor nominal. En este artículo, se propone el uso de un Controlador Repetitivo Impar de Alto Orden para el lazo de corriente del rectificador. Este controlador se diseña para obtener una señal sinusoidal de corriente y rechazar los armónicos impares introducidos en el sistema. Al atacar sólo los armónicos impares, el costo computacional de su implementación resulta muy similar al del controlador repetitivo convencional, con la ventaja de proporcionar robustez ante cambios en la frecuencia de la red. Los resultados experimentales muestran el alto desempeño del compensador aún cuando la frecuencia de la red se desvía del valor nominal de diseño.

\section{Palabras clave}

Rectificadores monofásicos, factor de potencia, distorsión armónica, calidad de potencia, control repetitivo.

\begin{abstract}
Controlled rectifiers are electronic power devices aimed at reducing the harmonic pollution in electrical networks caused by the power conversion process. The main goal is to obtain a sinusoidal shape current in phase with the voltage network. Although Proportional Integral controllers are widely used for the current control loop, they do not provide high performance results. On the contrary, Resonant and Repetitive Control are techniques with remarkable results on this area. However, their main drawback is the lost of performance due to frequency changes in the exogenous signal. In this work, the implementation of an Odd Harmonic High Order Repetitive Controller is proposed for the rectifier current loop. The odd harmonic characteristic of this compensator makes possible to obtain a computational burden that is very similar to the one obtained by conventional repetitive controllers with the advantage of increase the robustness against frequency variations. Experimental results show the high performance obtained even when the network frequency does not match the designed nominal frequency.
\end{abstract}

\section{Keywords}

PMW rectifiers, power factor correction, harmonic compensation, power quality, repetitive control. 


\section{INTRODUCCIÓN}

Los rectificadores controlados mediante señales de modulación de ancho de pulso (PWM) han surgido como una técnica orientada a la reducción de la contaminación armónica de la red eléctrica ([1], [2]) y como respuesta a los estándares cada vez más exigentes en materia de calidad de energía (ver normas IEC 61000 [3] e IEEE 519 [4]). El objetivo del control es obtener una señal de corriente sinusoidal en fase con el voltaje de la red. Para evaluar el desempeño de los rectificadores PWM se usan básicamente dos parámetros: la Distorsión Harmónica Total (THD) y el Factor de Potencia (PF), el primero da un estimado de la cantidad de armónicos presentes en la señal de corriente y el segundo mide el desfase que existe entre la señal de corriente y la señal de voltaje. De esta manera los rectificadores PWM buscan obtener un THD bajo y un FP cercano a la unidad.

El uso del control Proporcional Integral (PI) en el lazo de corriente de este tipo de rectificadores es común debido a lo sencillo de su diseño, su fácil implementación y los resultados favorables [5]. Este tipo de controladores logran un desempeño cercano a los límites requeridos por los estándares como IEEE 519, proporcionando algo menos del $5 \%$ de THD. Lograr mejores resultados, con muy bajo THD y FP unitario, es considerado como un valor agregado adicional en los diseños de rectificadores PWM. Sin embargo, la naturaleza del controlador PI no le permite proporcionar estos altos desempeños [6].

El control resonante [5], [7] y el control repetitivo (RC) [8]-[10] son estrategias diseñadas para el seguimiento y/o rechazo de señales periódicas. Con estas estrategias se pueden obtener resultados muy sobresalientes en los rectificadores PWM [5], [8]. Sin embargo, la principal desventaja de estas técnicas se basa en una importante pérdida del desempeño cuando la frecuencia de la señal externa varía [11]. Como forma de solucionar este problema se ha desarrollado el control repetitivo de alto orden (HORC) [12], [13]. Mediante esta técnica se logra obtener una mayor robus ${ }^{-}$ tez ante cambios en la frecuencia.

En este artículo se propone el uso de un controlador HORC impar para el lazo de corriente de un rectificador PWM. De esta manera, se diseña un compensador que, aprovechando la naturaleza de las perturbaciones, solo actúa sobre los armónicos impares. Con este diseño se logran dos características: 1) al ser impar el costo computacional se reduce, resultando muy similar a la carga computacional resultante con el control repetitivo convencional y 2) el sistema presenta robustez ante los cambios en la frecuencia de la red. Los resultados experimentales muestran el alto desempeño conseguido con este compensador al obtener un THD del 0,7\% y FP unitario con la ventaja adicional de que este desempeño se mantiene a pesar de los cambios de frecuencia esperados en la red eléctrica.

El artículo está organizado de la siguiente manera. En la primera sección se describe el modelo del rectificador PWM y se plantean los objetivos de control así como la estructura del controlador. Posteriormente se expone el control HORC impar para el lazo de corriente seguido de una sección descriptiva del lazo de voltaje. Finalmente se documentan los resultados experimentales y las conclusiones.

\section{RECTIFICADOR PWM}

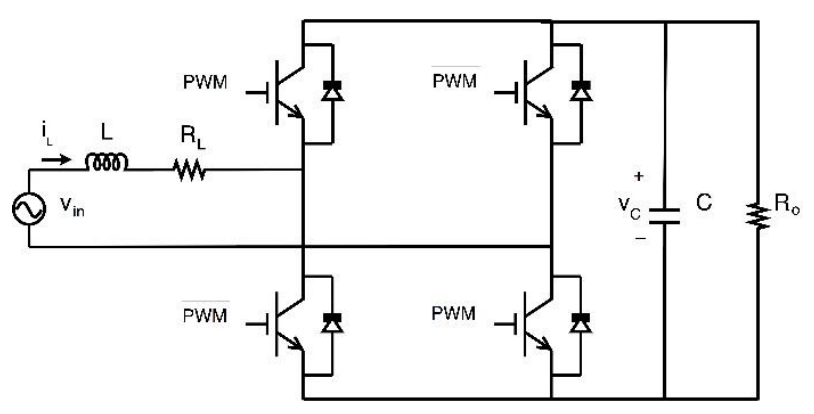

Fig. 1. Rectificador PWM. Fuente: Autores 


\subsection{Modelo del rectificador}

La Fig. 1 muestra la configuración básica de un rectificador PWM (Pulse Width Modulation). La tensión de la red se conecta a la entrada del rectificador y por medio del puente completo de interruptores controlados se obtiene un voltaje rectificado a la salida. La inductancia de entrada se utiliza para realizar la compensación de armónicos en la corriente en el lado de la red y el condensador se hace necesario para suavizar el voltaje de salida. El comportamiento dinámico del rectificador puede expresarse utilizando el modelo promediado a la frecuencia de trabajo del PWM, como se muestra en (1) y (2).

$$
\begin{gathered}
L \frac{d i_{L}}{d t}+R_{L} i_{L}=v_{i n}+d v_{c} \\
C \frac{d v_{c}}{d t}=d i_{L}-\frac{v_{c}}{R_{o}}
\end{gathered}
$$

en donde $L$ es la inductancia del rectificador, $R_{L}$ es la resistencia parásita de la inductancia, $i_{L}$ es la corriente en la inductancia, $C$ es el valor del condensador, $V C$ es el voltaje en el condensador, $R_{o}$ es la resistencia de carga, $v_{i n}=V_{i n} \sqrt{2} \sin \left(\omega_{n} t\right)$ es la fuente de voltaje de entrada y $\omega_{n}=$ $2 \pi / T_{p} \mathrm{rad} / \mathrm{s}$ es la frecuencia de la red. La acción de control $d$ está definida en el in- tervalo $[-1,1]$ y representa el valor promedio de la señal PWM enviada al sistema real.

\subsection{Objetivos de control}

El objetivo al controlar el rectificador es asegurar que la corriente a la entrada, $i_{L}(t)$, tenga una forma sinusoidal y se encuentre en fase con el voltaje de la red. Esto puede escribirse como $i_{L}^{*}=$ $I_{d}^{*} \sin \left(\omega_{n} t\right)$, en donde $x^{*}$ representa el valor de estado estacionario de la señal $x(t)$. De esta manera, se requiere que el sistema de control siga una señal sinusoidal de magnitud apropiada, $I_{d}$, y rechace las perturbaciones presentes en el sistema. Dentro de estas perturbaciones se encuentran señales de baja frecuencia y armónicos introducidos por las distorsiones presentes en el voltaje de la red. Las distorsiones en la forma de onda del voltaje de red generalmente se encuentran dentro del tercer y noveno armónico, todos ellos armónicos impares.

Adicionalmente, es necesario que el valor promedio del voltaje de salida (bus dc) se mantenga constante. Por lo tanto, se requiere que el sistema pueda soportar cambios en el voltaje de la red así como cambios en la carga del rectificador.

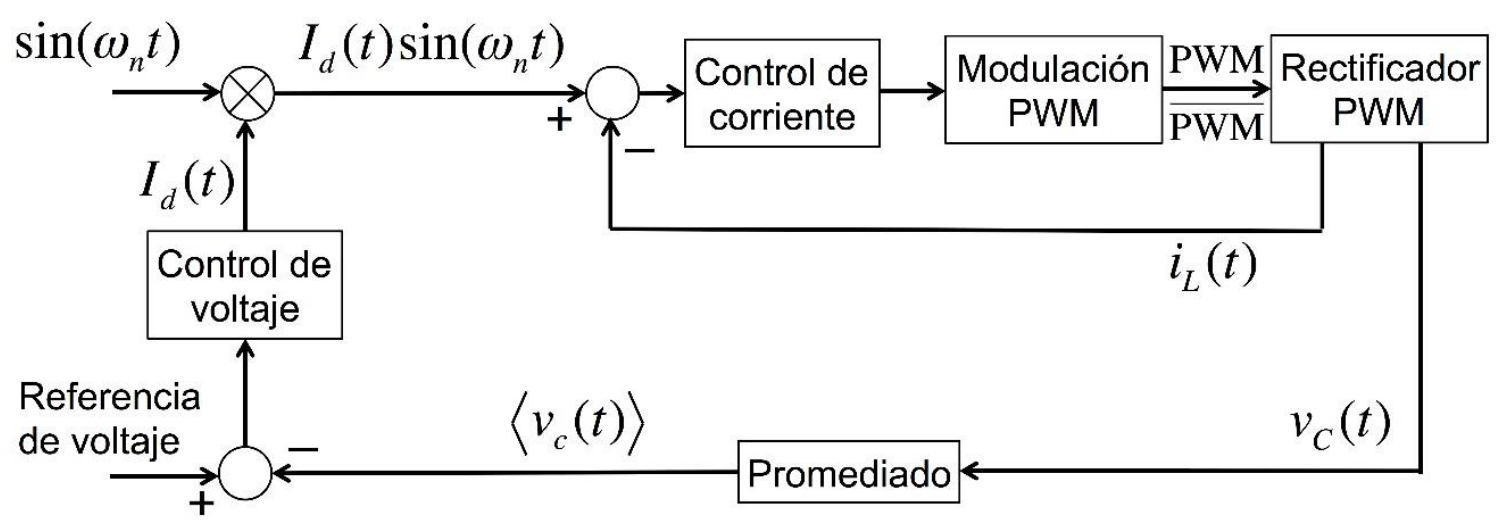

Fig. 2. Estructura de doble lazo del sistema de control. Fuente: Autores 
El esquema del sistema de control basado en los dos objetivos antes mencionados se muestra en la Fig. 2. El esquema se compone de dos lazos de control: un lazo interno o lazo de corriente encargado de asegurar la forma sinusoidal de la corriente de entrada y un lazo externo o lazo de voltaje diseñado para regular el voltaje de salida a un valor constante y proporcionar el balance de la potencia activa en el sistema. La salida de este último lazo proporciona la amplitud de la señal de referencia sinusoidal, $I_{d}$, para el lazo de corriente.

En este trabajo se propone para lazo de corriente un controlador repetitivo impar de alto orden, mediante esta propuesta se pretende compensar los armónicos impares presentes en el sistema cuando este está expuesto al voltaje de red y al mismo tiempo ofrecer robustez ante cambios en la frecuencia de la red.

Para el lazo de voltaje se utilizará un controlador Proporcional Integral (PI) convencional que permite realizar el balance de potencia activa y mantener constante el valor promedio del voltaje de salida.

La dinámica completa del sistema se descompone en dos partes: 1) una dinámica rápida dada en el lazo de corriente y 2) una dinámica lenta diseñada para el lazo del voltaje. Por lo tanto, para la convergencia del sistema completo se asume que el lazo de voltaje proporciona cambios muy lentos en la referencia de corriente y que el lazo de corriente opera tan rápido que su comportamiento dinámico no altera la evolución del lazo de voltaje. Para obtener este comportamiento es necesario diseñar adecuadamente el ancho de banda de cada lazo para lograr así una separación suficiente de las dos dinámicas.

\section{LAZO DE CONTROL DE CORRIENTE}

En esta sección se describe el controlador repetitivo impar de alto orden que se propone para el lazo de corriente. Inicialmente se describirá el control repetitivo convencional y la versión impar para finalmente diseñar el controlador de alto orden.

\subsection{Control repetitivo impar de alto orden}

El control repetitivo (RC) se ha consolidado como una estrategia eficaz en problemas de control que involucran el seguimiento y/o rechazo señales periódicas $[9,10]$. Haciendo uso de esta estrategia de control es posible seguir la señal sinusoidal de referencia en el lazo de control de co- $^{-}$ rriente y a la vez rechazar los armónicos introducidos por el voltaje de red. Para lograr esto, el RC incluye un generador de señales periódicas dentro de la estructura de control [13] (ver (3)).

$$
I(z)=\frac{\sigma W(z) H(z)}{1-\sigma W(z) H(z)}
$$

donde $W(z)$ es una función de retardo, $H(z)$ es un filtro pasabajos para proporcionar robustez en las altas frecuencias y $\sigma$, que toma el valor de $10^{-}-1$, determina si se actúa sobre todos los armónicos, $\sigma=1$, o únicamente sobre los armónicos impares, $\sigma=-1$.

Para obtener el modelo interno convencional utilizado en control repetitivo [14], se usa $H(z)=1, \sigma=1, W(z)=z^{-N}$, con $N=T_{p} / T_{s}$, siendo $T_{p}$ el periodo de la señal a seguir y/o rechazar y $T_{s}$ el periodo de muestreo del sistema de control. Este modelo proporciona ganancia infinita a la frecuencia fundamental y todos sus armónicos hasta el (N/2-1)-ésimo. Tener alta ganancia a las frecuencias seleccionadas permite que en lazo cerrado el sistema pueda seguir y/o rechazar señales con ese contenido frecuencial.

Por otro lado, un modelo interno con ganancia infinita solo en los armónicos impares [15], esto es, $\omega_{k}=2(2 \mathrm{k}-1) \pi / \mathrm{N}$ $\mathrm{rad} / \mathrm{s}$ con $\mathrm{k}=1, . ., \mathrm{N} / 2$, resulta de usar $H(z)=1, \sigma=-1, W(z)=z^{-N / 2}$. 
La Fig. 3 muestra la respuesta en frecuencia en magnitud para los modelos internos convencional e impar, sintonizados para una frecuencia fundamental de 60 $\mathrm{Hz}$ y un periodo de muestreo de $15 \mathrm{kHz}$, esto es, $\mathrm{N}=250$. Puede observarse que estos modelos ofrecen ganancia infinita de manera muy selectiva para la frecuencia fundamental y los armónicos correspondientes. Esta característica representa la principal desventaja de los modelos internos convencional e impar. Esto ocasiona que el control repetitivo convencional presente una gran pérdida de desempeño cuando la frecuencia de la señal externa se desvía de su valor nominal, incluso cuando los cambios son pequeños [11].

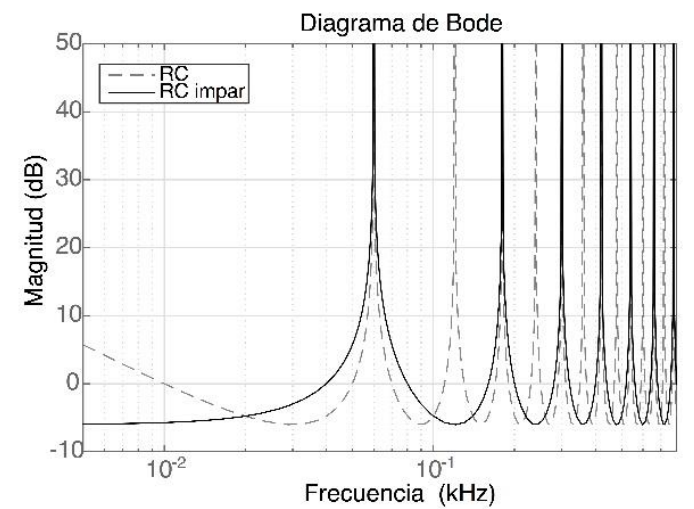

Fig. 3. Respuesta en frecuencia del modelo interno convencional y el modelo interno impar. Fuente: Autores

Finalmente, modificando la función de retardo $W(z)$, se puede lograr que el modelo interno proporcione alta ganancia para un intervalo mayor de frecuencias alrededor de la fundamental y los armónicos, por lo tanto ofreciendo mayor robustez ante los cambios en la frecuencia de la red. En consecuencia, se define $H(z)=1, \sigma=-1$, y la función de retardo como (4).

$$
W(z)=-1+\left(1+z^{-\frac{N}{2}}\right)^{M}
$$

donde $M$ define el orden del control repetitivo de alto orden (HORC). Con estas definiciones el modelo interno (3) resulta como se muestra en (5) (ver [11], [12]).

$$
I_{\text {odd }}(z)=\frac{1-\left(1+z^{-\frac{N}{2}}\right)^{M}}{\left(1+z^{-\frac{N}{2}}\right)^{M}}
$$

La Fig. 4 muestra la comparación de la respuesta en frecuencia de los modelos internos RC impar y HORC impar de segundo $(M=2)$ y tercer $(M=3)$ orden para una frecuencia fundamental de $60 \mathrm{~Hz}$ y un periodo de muestreo de $15 \mathrm{kHz}$. La Fig. 4 también muestra la ganancia obtenida para desviaciones de $59,5 \mathrm{~Hz}$ y $60,5 \mathrm{~Hz}$ al igual que para sus armónicos. Puede observarse, que para la misma desviación de frecuencia el modelo interno HORC impar $(M=2$ y $M=3)$ ofrece mayor ganancia que el modelo RC impar $(M=1)$. Esto hace que el sistema sea mas robusto ante las variaciones de la frecuencia y pueda mantener un mejor desempeño en condiciones de operación no ideales. Sin embargo, utilizar órdenes altos en el modelo interno puede llevar a la pérdida de la robustez debido a la elevada ganancia en los armónicos más altos. Esto se puede compensar usando una menor frecuencia de corte en el filtro $\mathrm{H}(\mathrm{z})$, pero esto a su vez, podría ocasionar una pérdida de desempeño al recortar la acción sobre los armónicos superiores. Por lo tanto, se debe realizar un balance entre el orden $M$ del modelo interno y el ancho de banda del filtro $\mathrm{H}(\mathrm{z})$ para obtener la robustez deseada tanto en desempeño como en estabilidad [16], [17].

Dado que las variaciones de frecuencia en el sistema eléctrico son pequeñas (en el contexto de sistemas eléctricos grandes) y que es deseable tener la menor distorción posible en la corriente de entrada del rectificador, el modelo interno impar de alto orden con $M=2$ (2ORC) se ha validado ex- $^{-}$ perimentalmente como el de mejor desempeño. 
El modelo interno impar resultante con el filtro pasabajos $H(z)$ y $M=2$ se puede ver en (6).

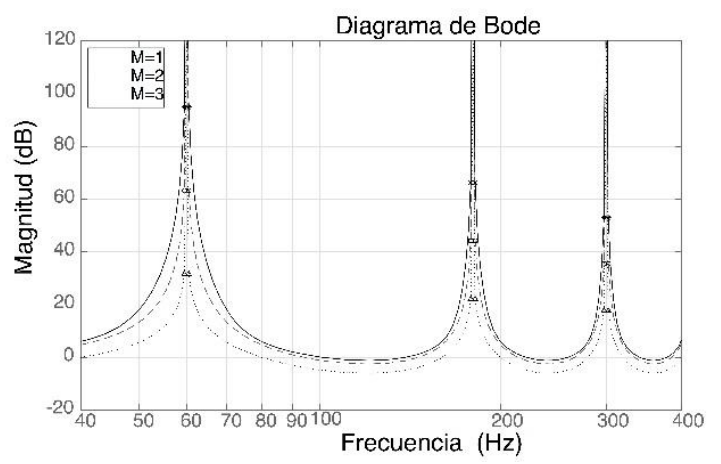

Fig. 4. Respuesta en frecuencia del modelo interno de alto orden. Fuente: Autores

$$
I_{2 o d d}(z)=\frac{-\left(2 z^{-\frac{N}{2}}+z^{-N}\right) H(z)}{1+\left(2 z^{-\frac{N}{2}}+z^{-N}\right) H(z)}
$$

Es importante notar que el modelo interno (6) presenta el mismo orden que el modelo usado en un controlador repetitvo convencional por lo tanto obteniéndose una carga computacional similar en la implementación.

\subsection{Estructura del controlador repetitivo}

El control repetitivo se implementa convencionalmente usuado la estructura plug-in (ver Fig. 5), en donde el modelo interno se adiciona a un controlador existente, $G_{c}(z)$, y además se incluye el filtro $G_{x}(z)$. El controlador $G_{c}(z)$, se utiliza para estabilizar la planta, $G_{p}(z)$, y proporcionar la atenuación de perturbaciones en el ancho de banda de trabajo del rectificador. Además, el compensador $G_{c}(z)$ debe proporcionar márgenes de robustez suficientemente buenos. El filtro $G_{X}(z)$, se diseña para asegurar la estabilidad del sistema en lazo cerrado.

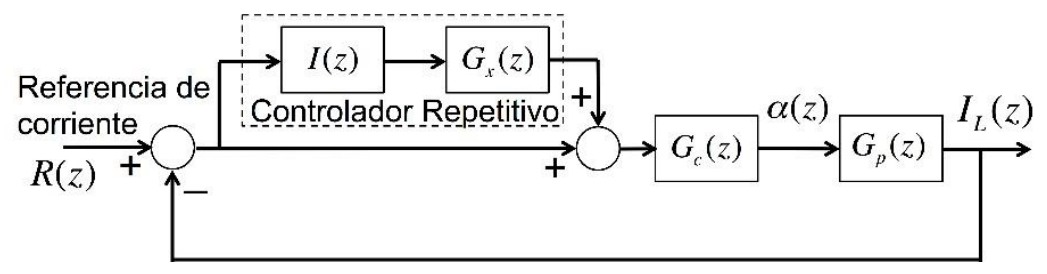

Fig. 5. Estructura del control repetitivo. Fuente: Autores

\subsection{Estabilidad}

El sistema de lazo cerrado de la Fig. 5 es estable si se cumplen las siguientes condiciones [18]:

1. El lazo cerrado sin el controlador repetitivo es estable, esto decir, la función de transferencia mostrada en (7) debe ser estable.

$$
T_{o}(z)=\frac{G_{c}(z) G_{p}(z)}{1+G_{c}(z) G_{p}(z)}
$$

2. $\left\|W(z) H(z)\left(1-T_{o}(z) G_{x}(z)\right)\right\|_{\infty}<1$, dónde $\mathrm{H}(\mathrm{z})$ y $G_{X}(z)$ se deben seleccionar para cumplir esta condición.

\subsection{Diseño del filtro H(z)}

El filtro $H(z)$ es utilizado para limitar el ancho de banda del control repetitivo y por lo tanto afecta el desempeño limitando el número de armónicos sobre los cuales el controlador tiene efecto. En general, la definición del ancho de banda de este filtro representa un balance entre estabilidad robusta y desempeño. Aunque se han propuesto técnicas de optimización para defi- 
nir el filtro $H(z)$ [19], de forma convencional, $H(z)$ se selecciona como filtro FIR (Respuesta Finita al Impulso) pasabajos de fase nula [20]. La selección de este filtro se mostrará en la sección de resutados experimentales.

\subsection{Diseño del filtro Gx(z)}

Para sistemas de fase mínima es común seleccionar $G_{x}(z)=k_{r}\left(T_{o}(z)\right)^{-1}$, mientras que para sistemas de fase no mínima se puede usar el diseño presentado en [14]. Finalmente, el valor de $k_{r}$ se selecciona como un compromiso entre robustez y desempeño en estado transitorio [21].

\section{LAZO DE VOLTAJE}

El lazo de voltaje se diseña para realizar el balance de potencia activa del rectificador y así proporcionar la magnitud de la corriente de referencia para el lazo de corriente. Dado que el voltaje a la salida del rectificador presenta rizado, es común introducir un filtro pasabajos para suavizar la señal. Esto se hace con el objetivo de evitar que el rizado se propague a la referencia de corriente $i_{d}(t)$ lo cual afectaría negativamente la distroción armónica de la corriente de entrada del rectificador $i_{L}(t)$. En este trabajo, se ha sustituido el filtro pasabajos por un filtro promediador sintonizado para $60 \mathrm{~Hz}$, como se muestra en (8).

$$
F(z)=\frac{1}{N} \frac{\left(1-z^{-N}\right)}{\left(1-z^{-1}\right)}
$$

Mediante este filtro se obtiene el voltaje promedio del voltaje de salida $V_{d c}=\left\langle v_{c}\right\rangle$.

La estructura del lazo de voltaje se presenta en la Fig. 6. Esta estructura presenta dos componentes: una acción de control de realimentación, $i_{d f b}$, formada por un compensador PI y una acción de prealimentación $i_{d f f}$ En el lazo de realimentación, el controlador PI mostrado en (9) regulará el valor promedio del voltaje de salida al valor desado con error igual a cero.

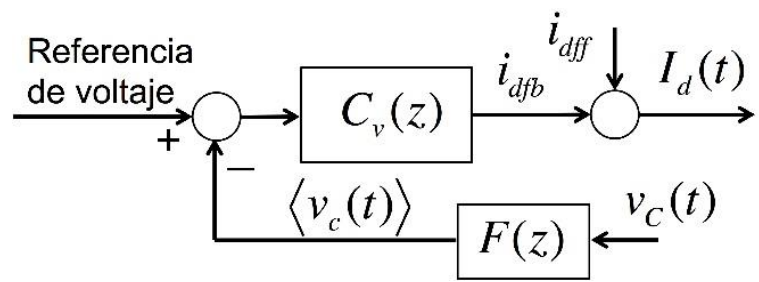

Fig. 6. Estructura del lazo de voltaje. Fuente: Autores

$$
C_{v}(z)=k_{i} \frac{T_{s}}{2} \frac{(z+1)}{(z-1)}+k_{p}
$$

Por el otro lado, la señal de prealimentación, $i_{d f f}$, se diseña para evitar que cambios abruptos en los niveles de voltaje produzcan transitorios con picos de corriente muy elevados. Esta prealimentación se calcula a partir del balance de potencia del rectificador en estado estacionario. De esta manera, el balance de la potencia activa en AC y la potencia en DC se muestran en (10).

$$
\frac{1}{2} V_{m} I_{m}=V_{d c} I_{d c}+P_{\text {pérdidas }}
$$

con $V_{m}=V_{\text {in }} \sqrt{2}$ el valor pico del voltaje de red, $I_{m}=I_{d}$ el valor pico de la corriente de entrada del rectificador, $V_{d c}=\left\langle v_{c}\right\rangle$ el valor promedio del voltaje en el condensador, $I_{d c}=\left\langle i_{L}\right\rangle$ el valor promedio de la $\mathrm{co}^{-}$ rriente de carga y $P_{\text {pérdidas }}$ las pérdidas en el rectificador. Con esto, el valor de la prealimentación puede calcularse como en (11)

$$
i_{d f f}=\frac{1}{2} \frac{V_{m} I_{m}}{V_{d c}}+I_{\text {pérdidas }}
$$

En donde $I_{\text {pérdidas }}$ es un factor que pondera las pérdidas en el rectificador.

Como resultado, la amplitud de la señal de referencia que se aplicará al lazo de corriente se obtiene mediante (12).

$$
I_{d}=i_{d f b}+i_{d f f}
$$




\section{RESULTADOS EXPERIMENTALES}

Esta sección presenta los resultados de experimentales del rectificador PWM. Se presentan resultados en condiciones nominales y resultados con variaciones en la frecuencia de la red.

\subsection{Configuración del rectificador}

El periodo de la señal de voltaje es $T_{p}=1 / 60$ s que corresponde con la frecuencia de la red eléctrica. El periodo de muestreo usado es $T_{s}=1 / 15000 \mathrm{~s}$ el cual corresponde con la frecuencia de trabajo de la señal PWM. Con esto se obtiene un periodo discreto de $N=T_{p} / T_{s}=250$ muestras.

La función de transferencia de la planta se obtiene a partir de (1). De esta manera, realizando la linealización con el cambio de variable $\alpha=d v_{c}$ se obtiene (13).

$$
G_{p}(s)=\frac{1}{s L+R_{L}}=\frac{I_{L}(s)}{\alpha(s)}
$$

A partir de la discretización de (13), como se muestra en (14),

$$
G_{p}(z)=\mathrm{Z}\left[\frac{1}{s L+R_{L}} \frac{1-e^{-T_{S}}}{s}\right]_{T_{S}}
$$

resulta un sistema de fase mínima. El valor de la inductancia es $L=600 \mathrm{mH}$ y su resistencia parásita $R_{L}=0,2 \mathrm{ohms}$. Para el lazo interno de corriente se diseña el controlador $G_{c}(z)=(6,293 z-6,283) /(z-0.998)$ el cual proporciona márgenes de robustez suficientes. El filtro $H(z)$ que proporciona un buen balance entre robustez y desempeño es $H(z)=-0,003871 z^{5}+0,03209 z^{3}+$ $0,1167 z^{2}+0,2207 z+0,2687+0,2207 z^{-1}+$ $0,1167 z^{-2}+0,03209 z^{-3}-0,003871 z^{-5}, \quad$ lo que determina un ancho de banda de 1440 $\mathrm{Hz}$.

Mientras que $G_{x}(z)=k_{r}\left(T_{o}(z)\right)^{-1} \quad \mathrm{y}$ $k_{r}=0.7$. El controlador PI del lazo del voltaje definido en (9) toma los valores $k_{p}=0,01 \mathrm{y}$ $k_{i}=0,7$.

Con fines de comparación se ha diseñado un control PI y un controlador repetitivo convencional impar en el lazo de corriente, manteniendo el mismo controlador en el lazo de voltaje. El controlador PI para el lazo de corriente está definido en (15).

$$
C_{i}(z)=k_{i i} \frac{T_{s} z}{(z-1)}+k_{i p}
$$

con $k_{i p}=0,2$ y $k_{i i}=0,45$. Para la sintonización de este controlador se buscó obtener el mayor ancho de banda posible evitando que la señal de control llegara a saturación o se tornara demasiado ruidosa. Por otro lado, el controlador repetitivo convencional RC utiliza el modelo interno (3) con $H(z)=$ $0,175 z+0.65+0,175 z^{-1}, \quad \sigma=-1$, $W(z)=z^{-N / 2}, N=250, G_{c}(z)$ definido como en $(9), G_{x}(z)=k_{r}\left(T_{o}(z)\right)^{-1}$ y $k_{r}=0,3$.

Los experimentos se realizaron conectando a la red eléctrica un autotransformador para así obtener un voltaje de entrada en el rectificador de $V_{i n}=20 \mathrm{Vrms}$. El rectificador monofásico es un convertidor electrónico de potencia configurable de marca Semikron. Los algoritmos de control se ejecutan utilizando un ordenador pentium con el núcleo de tiempo real XPCTarget de Mathworks. El algoritmo de control se ejecuta a la misma frecuencia de la señal PWM del rectificador que es de $15 \mathrm{kHz}$. La referencia del voltaje de salida se define como $V_{c}=36 \mathrm{~V}$ y la carga es una resistencia de 25 ohms. La Fig. 7 muestra la estrcutura de la plataforma experimental en donde puede verse el hardware adicional de generación y adquisición de señales. 


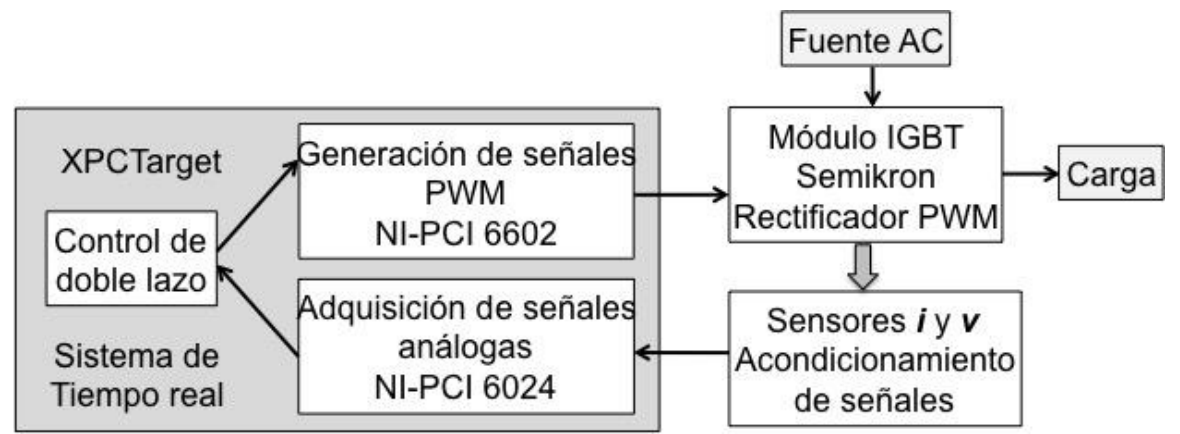

Fig. 7. Estructura de la plataforma experimental. Fuente: Autores

\subsection{Desempeño a frecuencia nominal}

La Fig. 8 muestra la respuesta de un rectificador de diodos no controlado con las mismas especificaciones utilizadas para el rectificador bajo estudio. Puede notarse la distorción y el contenido armónico de la corriente de entrada del rectificador, obteniéndose un valor de THD del 54,8\%.

Adicionalmente, la Fig. 8 muestra la señal de voltaje de entrada del rectificador, en donde puede observarse la distorsión armónica de la señal con contenido apreciable en el tercer y quinto componente. Es importante notar que este contenido armónico introduce perturbaciones en el lazo de corriente. Aunque las condiciones de la experimentación son variantes debido a la naturaleza de la red, todas las experimentaciones se recopilaron bajo condiciones muy similares.

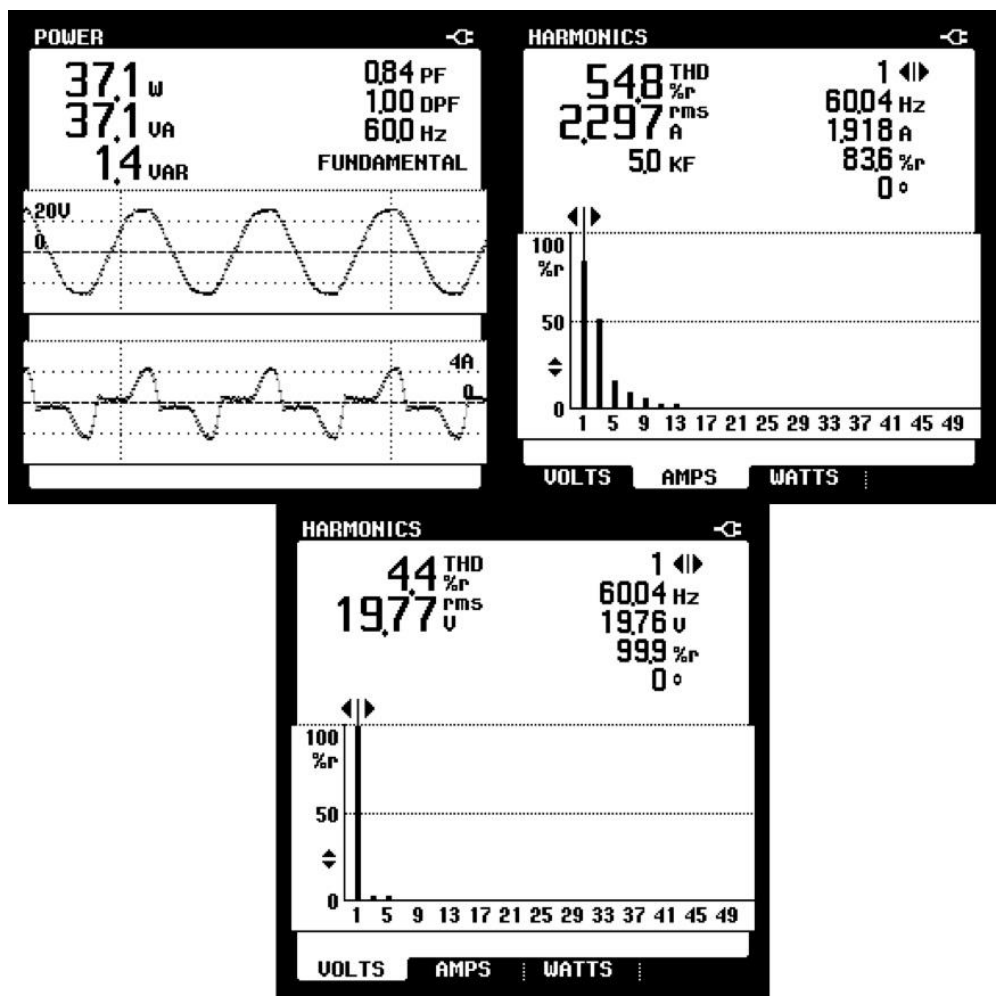

Fig. 8. Formas de onda producidas por un puente rectificador de diodos. Fuente: Autores 
Con fines de comparación se tienen tres diseños de controladores, controlador pro- $^{-}$ porcional integral PI, control repetitivo convencional impar $\mathrm{RC}$ y el controlador impar de alto orden 2ORC. Los diseños de los controlados PI, RC y 2 ORC se realizan para la frecuencia fundamental de $60 \mathrm{~Hz}$ como se describe en la sección anterior. Las Fig. 9, 10 y 11 muestran el desempeño de los diseños de los controladores PI, RC y 2ORC, respectivamente.
Por medio de estas figuras puede notarse que el controlador PI presenta el menor desempeño de los tres compensadores con un valor de THD de 3,7\% y se logra apreciar la presencia del tercer y quinto armónicos. Por otro lado, los dos controladores repetitivos exhiben un desempeño notablemente superior con una forma de onda de corriente muy cercana a la ideal. Esto muestra cómo el control repetitivo puede rechazar armónicos superiores que son introducidos por la distorsión presente en la señal de voltaje de una red real.

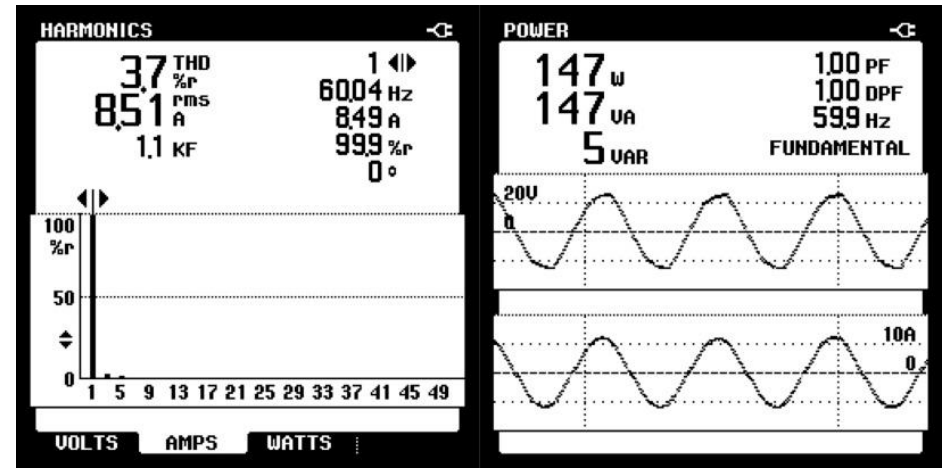

Fig. 9. Formas de onda y contenido armónico de la corriente para el controlador PI. Fuente: Autores

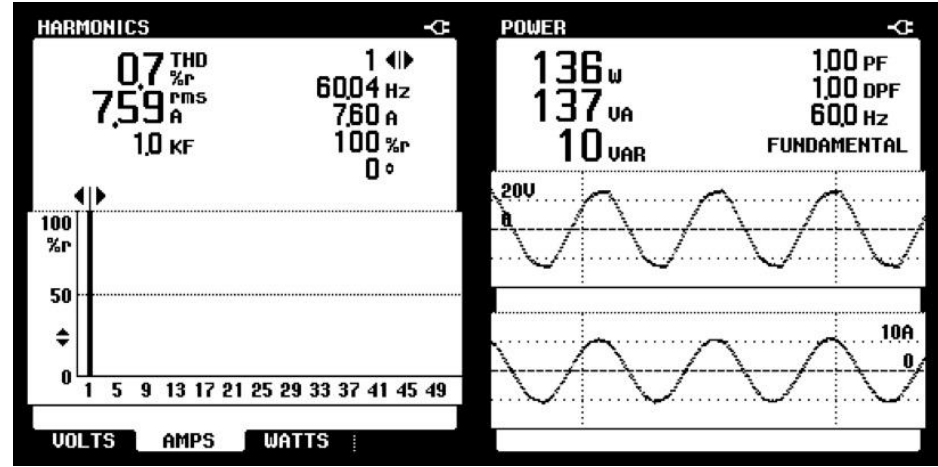

Fig. 10. Formas de onda y contenido armónico de la corriente para el controlador RC. Fuente: Autores

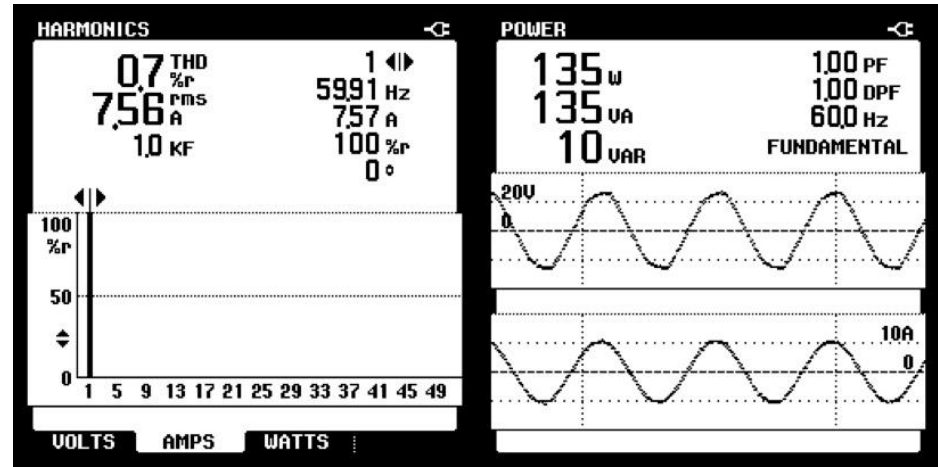

Fig. 11. Formas de onda y contenido armónico de la corriente para el controlador 2ORC. Fuente: Autores 


\subsection{Desempeño a frecuencia variable}

En este experimento se utilizó una fuente de frecuencia variable constituida por una UPS del fabricante PowerSun. Esta fuente se usó en lugar de la red eléctrica y se configuraron experimentos con frecuencias de $58 \mathrm{~Hz}$ y $61 \mathrm{~Hz}$.

La Fig. 12 y la Fig. 13 muestran el desempeño del controlador RC y 2ORC para las frecuencias mencionadas. Puede verse que el controlador RC pierde desempeño cuando existe diferencia entre la frecuencia a la que está sintonizado y la frecuencia de la fuente. Por otro lado, el control 2ORC ofrece mejor desempeño proporcionando así mayor robustez ante los cambios en la frecuencia de la red o desviaciones en la medición de la misma.

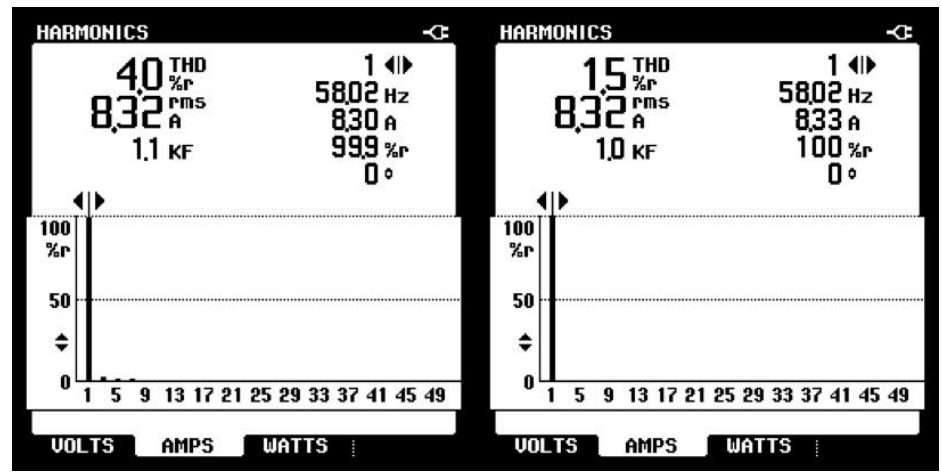

Fig. 12. Contenido armónico de la corriente para el controlador RC (izquierda) y controlador 2ORC (derecha) a una frecuencia de $58 \mathrm{~Hz}$. Fuente: Autores

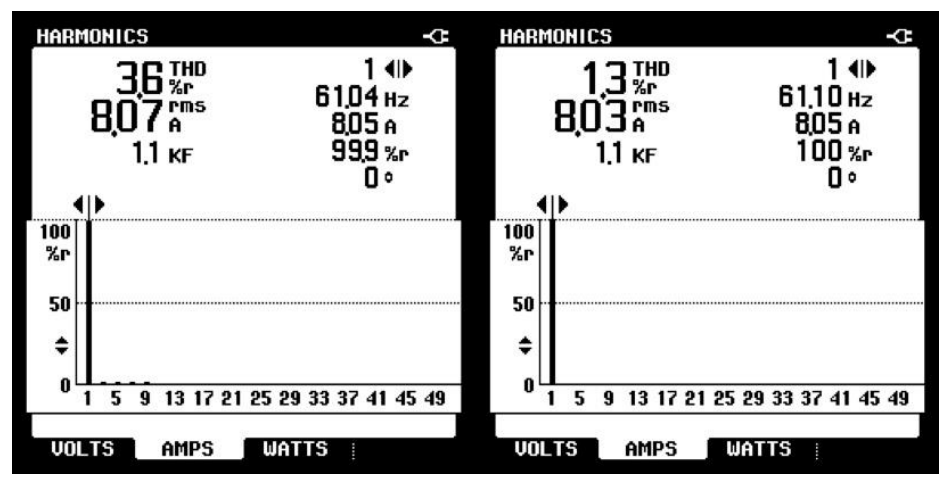

Fig. 13. Contenido armónico de la corriente para el controlador RC (izquierda) y controlador 2ORC (derecha) a una frecuencia de $61 \mathrm{~Hz}$. Fuente: Autores

\subsection{Variaciones en el voltaje de entrada}

Los siguientes experimentos muestran la regulación del voltaje de salida del rectificador cuando ocurren cambios en la amplitud del voltaje de la red. El voltaje de red cambia de 20 Vrms a 17 Vrms entre $t=25 \mathrm{~s} \mathrm{y} t=30 \mathrm{~s}$ mientras que la referencia del voltaje de salida es de $28 \mathrm{~V}$. La Fig. 14 muestra el cambio en el voltaje de la red y la respuesta en el voltaje de salida para el controlador PI, RC y 2ORC. Puede apreciarse que los cambios de amplitud de la red afectan el voltaje de salida pero después de superar el transitorio el sistema de control logra recuperar el nivel de voltaje requerido. Dado que el comportamiento dinámico del voltaje de salida está dominado por el lazo de voltaje, se puede observar que el desempeño es esencialmente el mismo para los tres controladores. 


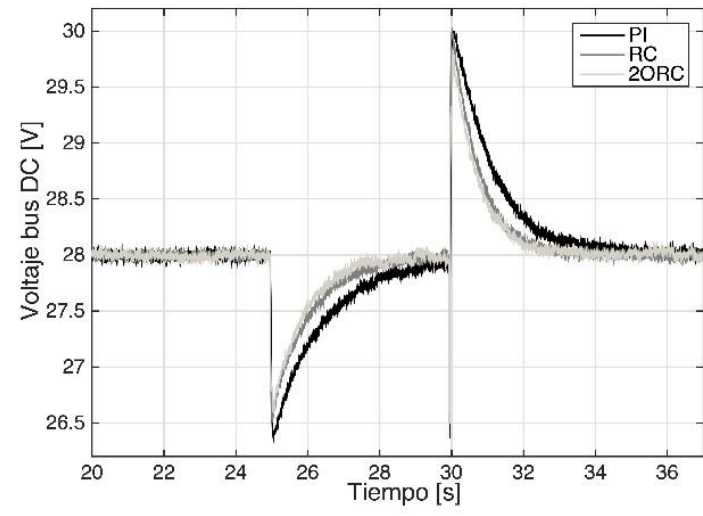

Fig. 14. Voltaje promedio de salida ante cambios en el voltaje de entrada. Fuente: Autores

\section{CONCLUSIONES}

En este artículo se ha propuesto el uso de un controlador repetitivo impar de alto orden para el lazo de corriente de un rectificador PWM. La validación experimental ha mostrado que con el compensador propuesto se logra un muy alto desempeño, obteniéndose así un THD de 0,7\% y FP unitario. Como ventaja principal frente a otros compensadores de alto desempeño, se tiene que el sistema de control presenta robustez ante los cambios en la frecuencia permitiendo la operación del rectificador en condiciones de frecuencia variable presentes en la red eléctrica.

A pesar de ser un compensador de alto orden, la característica impar de su modelo interno permite obtener gastos computacionales similares al del control repetitivo convencional.

\section{AGRADECIMIENTOS}

Este trabajo está financiado por la $\mathrm{Di}^{-}$ visión de Investigación Sede Bogotá (DIB) de la Universidad Nacional de Colombia bajo el Programa Nacional de Proyectos para el Fortalecimiento de la Investigación, la Creación y la Innovación en Posgrados de la Universidad Nacional de Colombia
2013 - 2015, con el proyecto Hermes 19348.

\section{REFERENCIAS}

[1] R. Etz, T. Patarau, and D. Petreus, "Comparison between digital average current mode control and digital one cycle control for a bridgeless PFC boost converter," in Design and Technology in Electronic Packaging (SIITME), 2012 IEEE 18th International Symposium for, 2012, pp. 211-215.

[2] J. R. Rodriguez, J. W. Dixon, J. R. Espinoza, J. Pontt, and P. Lezana, "PWM regenerative rectifiers: state of the art," Ind. Electron. IEEE Trans., vol. 52, no. 1, pp. 5-22, Feb. 2005.

[3] IEC, "IEC 1000-3-2" Electromagnetic Compatibility. Part 3: Limits- Sect.2: Limits for Harmonic Current Emission (Equipment Input Current 16A Per Phase)." 2014.

[4] IEEE, "IEEE Recommended Practices and Requirements for Harmonic Control in Electrical Power Systems, Revision of IEEE Std 519-1992.” pp. 1-112, 1993.

[5] F. Li, Y. Zou, W. Chen, and J. Zhang, "Comparison of current control techniques for single-phase voltagesource PWM rectifiers," in IEEE International Conference on Industrial Technology, 2008, pp. 1-4.

[6] Z. Shuai, A. Luo, C. Tu, and D. Liu, "New control method of injection" type hybrid active power filter," Power Electron. IET, vol. 4, no. 9, pp. 1051-1057, 2011.

[7] H.-S. Song, R. Keil, P. Mutschler, J. van der Weem, and K. Nam, "Advanced control scheme for a single-phase PWM rectifier in traction applications," in Industry Applications Conference, 2003. 38th IAS Annual Meeting. Conference 
Record of the, 2003, vol. 3, pp. 15581565 vol.3.

[8] C. Wang, Y. Zou, Y. Zhang, Y. Xu, X. She, and F. Li, "Research on the single-phase PWM rectifier based on the repetitive control," in Industrial Technology, 2008. ICIT 2008. IEEE International Conference on, 2008, pp. 1-6.

[9] R. W. Longman, "Iterative learning control and repetitive control for engineering practice," Int. $J$. Control, vol. 73, no. 10, pp. 930-954, 2000.

[10] M. Tomizuka, "Dealing with periodic disturbances in controls of mechanical systems," Annu. Rev. Control, vol. 32, no. 2, pp. 193-199, 2008.

[11] M. Steinbuch, "Repetitive control for systems with uncertain period-time," Automatica, vol. 38, no. 12, pp. 2103-2109, 2002.

[12] M. Steinbuch, S. Weiland, and T. Singh, "Design of Noise and PeriodTime Robust High Order Repetitive Control, with application to optical storage," Automatica, vol. 43, pp. 2086-2095, 2007.

[13] T. Inoue, "Practical repetitive control system design," in 29th IEEE Conference on Decision and Control, 1990, pp. 1673-1678 vol.3.

[14] M. Tomizuka, T.-C. Tsao, and K. Chew, "Analysis and Synthesis of Discrete-Time Repetitive Controllers," J. Dyn. Syst. Meas. Control, vol. 111, no. 3, p. 353, 1989.

[15] G. A. Ramos, R. Costa-Castelló, and J. M. Olm, "Analysis and design of a robust odd-harmonic repetitive controller for an active filter under variable network frequency," Control Eng. Pract., vol. 20, no. 9, pp. 895903, 2012.

[16] G. A. Ramos, R. Costa-Castelló, and J. M. Olm, Digital Repetitive Control under Varying Frequency Conditions, vol. 446. SpringerVerlag, 2013.

[17] G. Pipeleers, B. Demeulenaere, and S. Sewers, "Robust High Order Repetitive Control: Optimal Performance Trade Offs," Automatica, vol. 44, pp. 2628-2634, 2008.

[18] R. Griñó and R. Costa-Castelló, "Digital repetitive plug-in controller for odd-harmonic periodic references and disturbances," Automatica, vol. 41, no. 1, pp. 153-157, Jan. 2005.

[19] J. h. She, M. Wu, Y. h. Lan, and Y. He, "Simultaneous optimisation of the low-pass filter and statefeedback controller in a robust repetitive-control system," IET Control Theory Appl., vol. 4, no. 8, pp. 1366-1376, 2010.

[20] T.-C. Tsao and M. Tomizuka, "Robust Adaptive and Repetitive Digital Tracking Control and Application to a Hydraulic Servo for Noncircular Machining," J. Dyn. Syst. Meas. Control, vol. 116, no. 1, pp. 24-32, 1994.

[21] G. Hillerström and R. C. H. Lee, "Trade-offs in Repetitive Control," 1997. 